\title{
The interplay of conditional NCAM-knockout and chronic unpredictable stress leads to increased aggression in mice
}

\author{
Christine Kohl ${ }^{1,2}$, Orbicia Riccio ${ }^{1}$, Jocelyn Grosse ${ }^{1}$, Olivia Zanoletti ${ }^{1}$, Céline Fournier ${ }^{1}$, Stefanie M. Klampfl ${ }^{1}$, \\ Mathias V. Schmidt ${ }^{2}$, and Carmen Sandi ${ }^{1}$ \\ 'Laboratory of Behavioral Genetics, Brain Mind Institute, School of Life Sciences, École Polytechnique Fédérale de Lausanne, EPFL, Lausanne, \\ Switzerland and ${ }^{2}$ Max Planck Institute of Psychiatry, RG Neurobiology of Stress, Munich, Germany
}

\begin{abstract}
The neural cell adhesion molecule (NCAM) is a key regulator of brain plasticity. Substantial evidence indicates that NCAM is down-regulated by exposure to sustained stress and chronic stress seems to lead to increased aggression. In addition, constitutional NCAM deletion in mice has been shown to lead to increased intermale aggression and altered emotionality Forebrain-specific postnatal NCAM knockout was previously shown to impair cognitive function, particularly when animals were exposed to subchronic stress, but the effects on emotional and social behavior remain unclear. In this study, we investigated the potential interplay of a forebrain-specific postnatal NCAM deletion and exposure to different lengths of repeated stress (i.e. subchronic: 14 days; chronic: 29 days) on aggressive and emotional behavior. Our results show that postnatal deletion of NCAM in the forebrain leads to increased aggression and altered emotionality depending on the duration of stress, whereas conditional NCAM knockout has no basal impact on these behaviors. These findings support the involvement of NCAM in the regulation of emotional and aggressive behaviors, suggesting that diminished NCAM expression might be a critical vulnerability factor for the development of these behavioral alterations under repeated exposure to stress.
\end{abstract}

\section{Keywords}

Cell adhesion molecules, emotional behavior, gene-environment interaction, GxE, plasticity, social behavior, stress

\section{History}

Received 11 July 2013

Revised 27 August 2013

Accepted 29 August 2013

Published online 4 October 2013

\section{Introduction}

Neural cell adhesion molecule (NCAM), a prominent member of the immunoglobulin superfamily of adhesion molecules, is one of the best-characterized cell adhesion molecules (CAMs) with regard to its involvement in brain plasticity, remodeling and formation of synapses, and its ability to modulate synaptic characteristics (Aonurm-Helm et al., 2008; Cunningham et al., 1987). The expression levels of NCAM and its binding properties are crucial for neural remodeling and synaptic function. Disturbances in NCAM expression and dysregulation of NCAM signaling have been linked to various psychiatric disorders, such as depression, bipolar disorder and schizophrenia in humans (Brennaman \& Maness, 2010; Chekhonin et al., 2008; Cunningham et al., 1987; Poltorak et al., 1995; Vawter et al., 1999; for review, see Sandi \& Bisaz, 2007), as well as with changes in emotional and social behaviors in animal models (Conboy et al., 2010; Stork et al., 1999, 2000). Of specific interest is pathological aggression, a

Correspondence: Prof. Carmen Sandi, Laboratory of Behavioral Genetics, Brain Mind Institute, School of Life Sciences, École Polytechnique Fédérale de Lausanne (EPFL), 1015 Lausanne, Switzerland. E-mail: carmen.sandi@epfl.ch key symptom in mental disorders (such as antisocial personality disorder and borderline personality disorder) which is also characteristic of many other psychiatric diseases, such as mood disorders and schizophrenia (Haller \& Kruk, 2006; Neumann et al., 2010; Veenema \& Neumann, 2007). Constitutive knockout of NCAM has been shown to increase intermale aggression (Stork et al., 1997). Furthermore, a deficiency in polysialic acid (PSA) during early development, which renders NCAM less adhesive, has been linked to changes in social motivation and increased aggression (Calandreau et al., 2010).

The interaction of genetic risk factors and environmental conditions appears to be a necessary requirement for the development of personality and psychiatric disorders. In particular, exposure to chronic or severe stress increases the incidence of such disorders (de Kloet et al., 2005; Lupien et al., 2009; Wingenfeld et al., 2010), and can act as a trigger for impaired social behaviors, especially aggression (Cordero et al., 2012; Craig, 2007; Craig \& Halton, 2009; Marquez et al., 2013; Mineur et al., 2003; Neumann et al., 2010; Wood et al., 2003). While brief periods of controllable stress result in mostly adaptive physiological and behavioral changes, chronic exposure to stress has been shown to have deleterious effects on several brain structures involved in regulating social behavior, including the hippocampus, prefrontal cortex and amygdala (McEwen, 2002; 
Nunes et al., 2009; Sandi, 1998, 2004). A large body of evidence implicates the differential expression of CAMs, particularly NCAM, in the mediation of stress effects on brain and behavior. Due to their location both pre-and postsynaptically and their function in synapse maturation and specialization (Dalva et al., 2007; Shapiro et al., 2007), CAMs might represent ideal candidate molecules for stress-induced plasticity and circuit remodeling in the brain (Sandi, 2004). Chronic stress has been shown to affect the expression of NCAM, among others, in multiple brain regions, including the hippocampus, prefrontal cortex and amygdala (Sandi, 2004; Sandi et al., 2001; Venero et al., 2002). Furthermore, altered expression of different CAMs has been associated with the modulation of social behaviors, including aggression (Calandreau et al., 2010; Dahlhaus \& El-Husseini, 2010; Hines et al., 2008; Kohl et al., 2013; Stork et al., 1997). Additionally, subchronic unpredictable stress has been shown to induce depression-like behavior and cognitive deficits in conditional NCAM-knockout mice, but not in wild-type littermates, rendering NCAM-deficient mice more vulnerable to stress (Bisaz \& Sandi, 2011). Therefore, we reasoned that a similar stress-related, time-dependent effect could be observed for NCAM-dependent social behaviors.

We aimed to determine whether there was an interaction of conditional NCAM knockout and the extent of exposure to repeated stress (i.e. subchronic versus chronic) on aggression. To test this hypothesis, we employed a "gene-environment" experimental design to determine if conditional NCAM-KO mice, lacking postnatal forebrain NCAM expression, are more vulnerable to the development of an aggressive phenotype following subchronic and/or chronic stress than their wild-type littermates.

\section{Material and methods}

\section{Animals}

All experiments were conducted on age-matched (13-15 months) conditional NCAM-deficient male mice (NCAMff + , further referred to as "NCAM-cKO") and their wild-type littermates (NCAMff-, further referred to as "WT") derived from in-house breeding at the animal facility of the École Polytechnique Fédérale de Lausanne (EPFL). The generation of these conditional NCAM-deficient mice has been previously described (Bukalo et al., 2004). Briefly, homozygous NCAM-floxed females were bred with homozygous NCAMfloxed males expressing cre-recombinase under the control of the promoter of the $\alpha$-subunit of the calcium-calmodulindependent protein kinase II ( $\alpha$ CaMKII). The offspring were homozygous for the NCAM-floxed alleles, with approximately $50 \%$ carrying the $\alpha$ CaMKII-cre transgene; the remainder were wild-type littermates. All mice were backcrossed for more than 10 generations to the C57BL/6J background.

All animals were housed in groups of two to four in standard plastic cages $(19 \times 27 \times 13 \mathrm{~cm})$ on a $12 \mathrm{~h} \mathrm{light/dark}$ cycle (lights on at 07:00h). Temperature and humidity in the animal housing room were maintained at $23^{\circ} \pm 1$ and $50 \% \pm 15 \%$, respectively. Food and water were available ad libitum. All procedures described were conducted according to the Swiss National Institutional Guidelines of Animal Experimentation and were approved through a license issued by the Cantonal Veterinary Authorities (Vaud, Switzerland). All efforts were made to minimize animal suffering during the experiments.

\section{Experimental design}

\section{Experiment 1}

Male animals of both genotypes $\left(n_{(\mathrm{WT})}=8 ; n_{(\mathrm{NCAM}-\mathrm{cKO})}=12\right)$ were characterized for anxiety-like and exploratory behavior as well as aggression, to investigate the possible influence of the conditional forebrain NCAM deletion under basal conditions.

After transfer to the animal facility for behavioral testing, the animals were given 2.5 weeks to habituate to the new facility. All animals were then characterized for anxiety-like behavior in the elevated plus maze (EPM). Subsequently, we tested the animals for exploratory and emotional behavior in the open field (OF) test, followed by the bedding preference (BP) test to verify no alterations in olfaction and the resident-intruder (RI) test to investigate aggression-like behavior. Animals were allowed to rest 1-2 days between each test. Seven days after the last test, all animals were sacrificed.

\section{Experiment 2}

To investigate whether exposure to stress interacts with the gene knockout on the development of aggressive behaviors, a second batch of animals $\left(n_{(\mathrm{WT})}=9 ; n_{(\mathrm{NCAM}-\mathrm{cKO})}=13\right)$ were subjected first to an unpredictable stress paradigm for 14 days and then to another period of unpredictable stress that was administered for 15 days. The first stress period was defined as "subchronic" stress, while after both unpredictable stress periods the level of stress exposure was defined as "chronic" (Bisaz \& Sandi, 2011). Anxiety-like behavior, exploratory behavior and aggression were tested after each stress period to investigate if the animals were differentially affected depending on the duration of the stress.

After the transfer from the breeding facility, the mice were allowed to habituate to the animal facility before phenotyping and were then characterized using the EPM for anxiety-like behavior and the BP test for olfaction. Four days later, all animals were subjected to the first period of unpredictable stress (further referred to as subchronic stress). On the day after the last stressor, the animals were tested for exploration and emotional behavior in the OF test; 2 days later they were tested in their home cage using the RI test for aggressive behavior towards an unknown intruder. The second period of unpredictable stress (further referred to as chronic stress) began on the day after the RI test, and lasted for 15 days. Again, all animals were tested in the OF and RI test and sacrificed 8 days after the RI test. A schematic overview of both experiments is shown in Figure 1.

\section{Subchronic and chronic unpredictable stress}

In experiment 2, all animals of both genotypes underwent the subchronic and chronic unpredictable stress paradigm after a habituation phase of 2.5 weeks following arrival in the EPFL behavioral phenotyping facility. The subchronic unpredictable stress period was defined by a length of 14 days and chronic unpredictable stress was defined by the two 
(A) single-housing

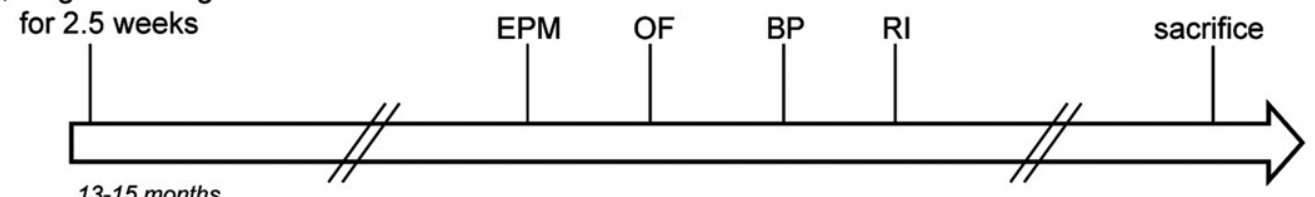

13-15 months

(B) single-housing for 2.5 weeks

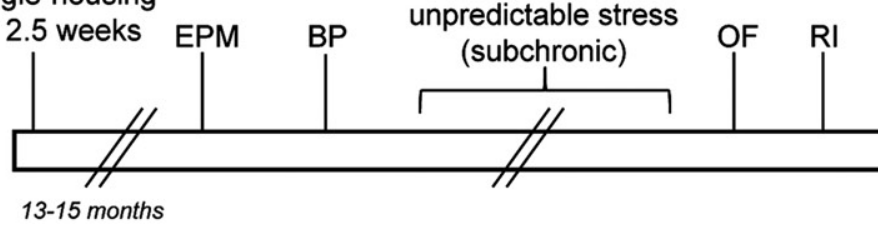

14 days npredictable stress (subchronic)

Figure 1. Experimental timeline. (A) Experiment 1: Basal characterization. All animals $\left(n_{(\mathrm{WT})}=8 ; n_{\text {(NCAM-cKO) }}=12\right)$ were tested for anxiety-like behavior in the elevated plus maze (EPM), exploratory and emotional behavior in the open field test(OF), olfaction using the bedding preference test (BP) and aggression in the resident-intruder test (RI). The inter-test interval was 1-2 days. (B) Experiment 2: Influence of conditional NCAM-knockout on behavior after subchronic and chronic stress. Animals $\left(n_{(\mathrm{WT})}=9 ; n_{(\mathrm{NCAM}-\mathrm{cKO})}=13,13-15\right.$ months old $)$ were characterized in the EPM and in the BP test. Four days later, all animals were subjected to the subchronic stress and subsequently tested for exploration, emotional behavior, and aggressive behavior. For chronic stress, a second period of stress ( 15 days) was started immediately after. Again, all animals were tested in the OF and RI test and sacrificed.

consecutive stress periods of 14 and 15 days, respectively. The stress protocol involved the exposure to one stressful situation per day at an unpredictable time point between 8 a.m. and 4 p.m. for a total duration of 14-15 consecutive days for each stress period. The following stressors were randomly distributed over time: tail suspension for 6 minutes; 4 hours exposure to soiled, damp sawdust; $40^{\circ}$ inclination of the home cage for 8 hours; immobilization in a plastic tube for 1 hour; transfer to a cage of conspecifics for 4 hours; two inescapable foot shocks at $0.4 \mathrm{~mA} ; 30$ minutes exposure to intensive light (600 Lux); encounter of conspecific defeat (see Wagner et al., 2013; animals were returned to the home cage after the defeat); 30 minutes of cold exposure at a temperature of $16^{\circ} \mathrm{C}$; 2 days of an inverted light:dark cycle; and a 6 minutes forced swim task. Body weight was monitored before subjecting the animals to stress, after the first period of stress (subchronic) as well as following the second period of stress (chronic).

\section{Behavioral analyses}

In all behavioral tests, video-recording (MediaCruise, Canopus Co., Ltd., Kobe, Japan) was performed, and an automatedtracking system (Ethovision 3.1, Noldus IT) was used.

\section{Elevated plus maze}

The elevated plus maze (EPM) was used to assess the animals' baseline anxiety-like behavior. The apparatus, made of black PVC with white inlays, consisted of an elevated platform (71 cm above the ground) with two opposing open arms $(30 \times 5 \mathrm{~cm})$, two opposing closed arms $(30 \times 5 \times 14 \mathrm{~cm})$ and a central platform $(5 \times 5 \mathrm{~cm})$ in the shape of a plus sign. Light conditions were set to $14-15$ lux in the open arms and 3-4 lux in the closed arms. All mice were individually placed onto the maze facing one of the closed arms. Animals were allowed to explore the apparatus for 5 minutes. Parameters of interest were the percentage of time spent in the open arms, the latency to enter the open arms and the distance moved in the whole apparatus. The maze was cleaned with $7 \%$ ethanol after every run.

\section{Bedding preference test}

As olfaction is crucial for social behavior, all animals were tested in the bedding preference test (BP), performed in the dark phase under red light conditions (after 7 p.m.). Two transparent perforated plastic drinking bottles $(6 \mathrm{~cm}$ in diameter, $14 \mathrm{~cm}$ high) were placed on opposite sides of the open field arena described below. One bottle was filled with a mixture of bedding from cages with untreated, adult male $\mathrm{C} 57 \mathrm{Bl} / 6$ mice, whereas the other contained clean bedding. The animals were introduced to the arena near the wall and allowed to freely explore it for 10 minutes. The arena was cleaned with $7 \%$ ethanol after every run. All videos were manually scored and analyzed by an experimenter blind to condition for the percentage of time spent sniffing each bottle.

\section{Open field test}

We performed the open field test (OF) to investigate the animals' emotional and exploratory behavior at baseline condition or following subchronic and chronic stress on the day after the last stressor. The test was conducted in a rectangular open arena $(50 \times 50 \mathrm{~cm}, 40 \mathrm{~cm}$ high). The animal was placed near the wall and allowed to freely explore the apparatus for 30 minutes. The open field was virtually divided in two parts: a center zone in the middle of the arena $(25 \times 25 \mathrm{~cm})$ and an outer zone along the walls of the arena. The parameters analyzed were the total distance traveled during the test, the percentage of time spent in the center zone, and the changes in those measures between the two testing sessions in experiment 2 . The open field arena was cleaned with $7 \%$ ethanol after every test.

\section{Resident-intruder test}

Seven days after OF testing in experiment 1 and two days after the $\mathrm{OF}$ test in experiment 2, all animals were evaluated for aggressive behavior using the resident-intruder (RI) paradigm. The test was conducted in the dark phase (after 7 p.m.), under red light conditions. An unfamiliar Balb/c mouse (intruder) 
was introduced to the experimental animal's home-cage for 2 trials (10 minutes each) with an inter-trial interval of 2 minutes. All intruders were matched for anxiety based on EPM results for each experimental group, as well as for body weight for the respective resident. All videos were manually scored after test completion by an experimenter blind to the conditions. Offensive behavior was scored in terms of attack duration and tail rattling and expressed as the percentage of the total test duration. For experiment 2, we additionally calculated the change in offensive behavior between the two testing sessions (i.e. the one following subchronic to the one following chronic stress conditions).

\section{Statistics}

All results are shown as mean + SEM and were analyzed using the software SPSS 17.0 (SPSS Inc., Chicago, IL). Comparisons of the two groups in experiment 1 and comparison of EPM data and changes in aggressive behaviors in experiment 2 were performed using independent $t$-tests, while comparisons of two parameters within one group were analysed with paired $t$-tests (bedding preference test: comparison between male bedding and clean bedding interaction). In experiment 2, comparisons of the groups following subchronic and chronic stress were conducted using a repeated measures ANOVA with genotype as betweensubjects factor and stress duration as within-subjects factor. Independent Student $t$-tests were performed as post-hoc tests when appropriate. In all analyses, the level of statistical significance was set at $p<0.05$.

\section{Results}

\section{Conditional forebrain NCAM-deletion does not impact body weight changes throughout subchronic and chronic stress}

Body weight changes were monitored throughout both periods of stress and compared to the initial body weight of animals prior to being subjected to any stress. Two-way repeated measures ANOVA revealed a significant effect of stress duration, but no genotype or stress duration by genotype interaction effects were detected (data not shown; stress effect $-F_{(2,19)}=41.831, p<0.001$; genotype effect $-F_{(1,20)}=$ $0.129, p>0.05$; interaction $\left.-F_{(2,19)}=1.050, p>0.05\right)$.

\section{Conditional forebrain NCAM-deletion does not affect anxiety-like behavior under basal conditions}

All animals were first characterized in the EPM for anxietylike behavior. In the two experiments performed, we did not detect differences between the two genotypes for the percentage of time spent in the open arms (exp.1: $t_{(18)}=1.266, p>0.05$; exp.2: $t_{(18.316)}=0.928, p>0.05$; data not shown), the latency to enter the open arms (exp.1: $t_{(18)}=-0.503, p>0.05$; exp.2: $t_{(21)}=-1.349, p>0.05$; data not shown) or the distance traveled in the apparatus (exp.1: $t_{(18)}=1.410, p>0.05$; exp.2: $t_{(18.085)}=0.123, p>0.05$; data not shown).

\section{Conditional forebrain NCAM-deletion does not alter olfaction}

Animals were tested for olfaction to exclude any influence of the genotype that could underlie potential behavioral abnormalities in social behaviors. Under basal conditions (exp. 1), all mice preferred the soiled bedding over the clean bedding (Figure 2A; paired $t$-tests, WT $t_{(7)}=-3.666$, $p<0.01$; NCAM-cKO $\left.t_{(11)}=-3.381, p<0.01\right)$ and no differences were found between the experimental groups (Figure 2A; male bedding $t_{(10.077)}=1.565 . p>0.05$; clean bedding $\left.t_{(18)}=0.139, p>0.05\right)$. Similarly, all animals in the stress study (exp. 2) showed a preference for the soiled bedding over the clean bedding (Figure 2B; paired $t$-tests, WT $t_{(8)}=-5.436, p<0.01 ; \quad$ NCAM-cKO $t_{(12)}=-5.757$, $p<0.001$ ), and no genotype differences were observed (Figure 2B; male bedding $t_{(20)}=-0.517, p>0.05$; clean bedding $\left.t_{(20)}=-0.431, p>0.05\right)$.
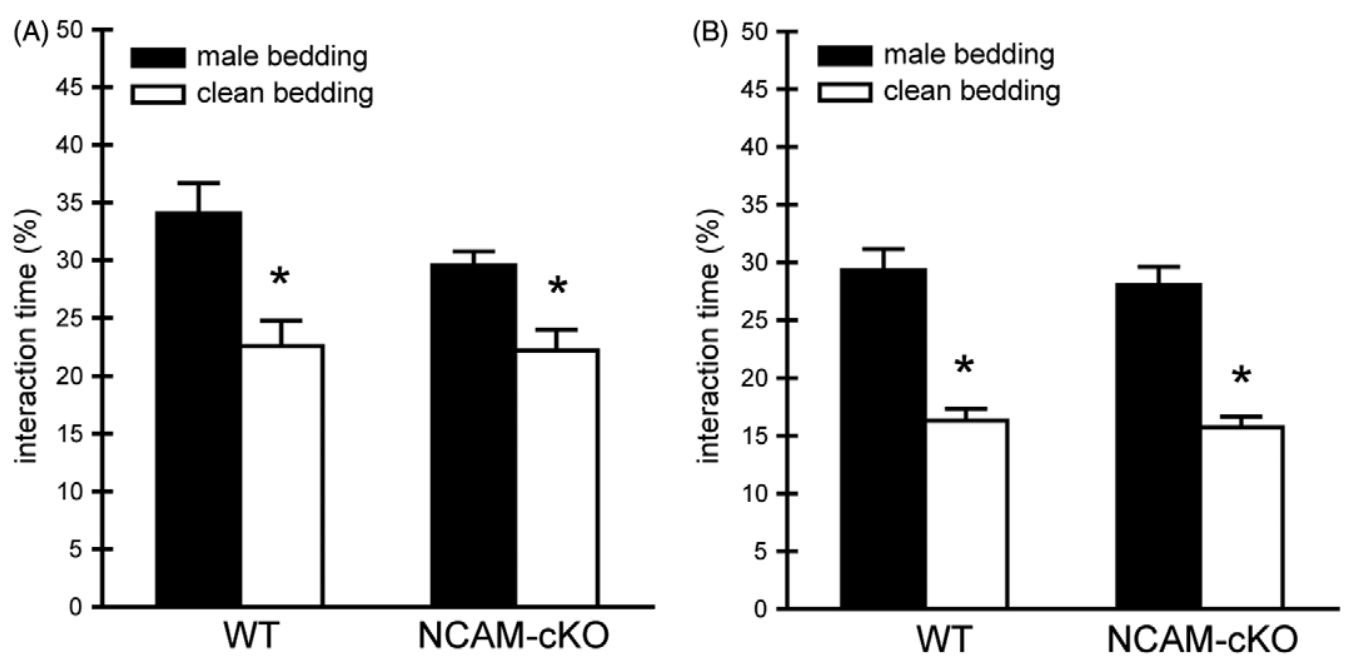

Figure 2. Intact olfaction. (A) Experiment 1. Animals of both genotypes were tested for olfaction under basal conditions. All mice preferred the soiled, male bedding over the clean bedding. No genotype differences were detected in the interaction pattern. (B) Experiment 2. All animals showed a significantly higher percentage of interaction time with the male bedding in comparison to the clean bedding. No genotype differences were observed. ${ }^{*} p<0.01$ versus clean bedding. 


\section{Conditional forebrain NCAM-deletion affects anxiety-like behavior following stress exposure}

To investigate the influence of the conditional knockout of NCAM on locomotion and exploratory behavior, as well as emotional behavior in a new environment, all mice were subjected to the OF test. Under baseline conditions (exp. 1), we did not observe any effect of genotype on the distance travelled (Figure $3 \mathrm{~A} ; t_{(18)}=-0.583, p>0.05$ ) or on the time spent in the center zone of the apparatus (Figure 3B; $\left.t_{(18)}=-1.186, p>0.05\right)$. Following subchronic and chronic unpredictable stress (exp. 2), two-way repeated measures ANOVA showed a significant effect of stress duration on the distance travelled, but failed to detect any significant genotype differences or interaction between the factors (Figure 3C and $\mathrm{E}$; stress effect $-F_{(1,20)}=18.201, p<0.001$; genotype effect $-F_{(1,20)}=3.109, p>0.05$; interaction $-F_{(1,20)}=2.675$, $p>0.05)$. Regarding the percentage of time spent in the center zone of the OF arena, two-way repeated measures ANOVA revealed significant effects of stress duration and genotype, but no interaction between these factors (Figure 3D and F; stress effect $-F_{(1,20)}=5.812, p<0.05$; genotype effect $F_{(1,20)}=7.532, \quad p<0.05 ; \quad$ interaction $-F_{(1,20)}=2.897$, $p>0.05)$.

\section{Conditional forebrain NCAM-deletion results in increased aggression after chronic stress}

In the RI test, we analyzed displays of offensive behavior towards an unknown intruder. There were no significant differences between NCAM-cKOs and WTs under basal conditions (exp. 1; Figure 4A; $t_{(18)}=0.963, p>0.05$ ). Following subchronic and chronic stress, two-way repeated measures ANOVA indicated a significant interaction of stress duration and genotype (Figure 4B and C; stress effect $F_{(1,20)}=3.946, p>0.05$, genotype effect $-F_{(1,20)}=4.084$, $p>0.05$, interaction $\left.-F_{(1,20)}=6.855, p<0.05\right)$. Compared to WT mice, NCAM-cKOs showed significantly increased offensive behavior towards intruders after chronic stress $\left(t_{(20)}=0.140, p<0.01\right)$. When calculating the respective change of offensive behavior from RI1 to RI2 (subchronic to chronic stress) using an independent $t$-test, NCAM-cKOs showed a significant increase in aggressive behavior compared to WTs (Figure 4D; $t_{(20)}=2.618, p<0.05$ ).

\section{Discussion}

In the present study, we investigated the impact of a forebrainspecific conditional knockout of NCAM on aggressive behavior in mice, at baseline conditions and after two subsequent periods of repeated unpredictable stress. Under basal conditions and following subchronic exposure to stress (i.e. during 14 consecutive days), there were no differences in aggressive behavior between the two genotypes. However, following chronic exposure to stress (i.e. 29 days), NCAMcKO mice, but not their wild-type littermates, showed increased offensive behavior towards an unknown intruder. Alterations in emotional but not exploratory behavior were observed in NCAM-cKO mice following stress exposure.

As shown by Stork and colleagues (Stork et al., 1997), constitutive NCAM-KOs on the same genetic background display increased inter-male aggression towards an unfamiliar intruder in their home cage. In our study, no effect of the conditional NCAM-KO genotype was observed in the basal and subchronic stress condition, and the effects of the conditional NCAM knockout only appeared after a stronger environmental challenge (i.e. chronic stress exposure), highlighting the importance of gene $\times$ environment interaction. A broad variety of studies have indicated a gene $\times$ environment interaction in the development of antisocial and especially aggressive behavior (Moffitt, 2005; Newman et al., 2005; Rutter \& Silberg, 2002; Rutter et al., 2006). While many findings concerning the development of antisocial or aggressive behaviors have been based on early life adverse experiences (Caspi et al., 2002, 2003; Frazzetto et al., 2007), we show here that the combination of altered postnatal NCAM expression and prolonged stress in adulthood results in altered aggressive behavior. As chronic stress exposure in rats has been shown to lead to enhanced aggressive behavior towards cage mates (Wood et al., 2008) and NCAM expression has been shown to decrease after chronic stress exposure (Mineur et al., 2003; Sandi et al., 2005; Venero et al., 2002), this result further supports the possible role of decreased NCAM expression in the development of abnormal aggression. In our study, chronic, but not subchronic, stress induced differences in aggressive behavior, thus rendering the duration of the stress an important factor in the interaction of NCAMdeletion with environmental challenges. This might specifically account for aggression-related behavior because subchronic stress has been previously shown to be sufficient to induce differences in depression-like behavior between wild types and NCAM-cKOs (Bisaz \& Sandi, 2011).

Exploration was not altered in stressed NCAM-cKOs when compared to WT littermates. Previously, increased exploratory behavior in the $\mathrm{OF}$ test has been associated with constitutive NCAM-KO (Aonurm-Helm et al., 2008). Logically, a constitutive knockout of the NCAM gene (i.e. a total deletion of NCAM also during the entire life span including development) should have a stronger impact on behavioral characteristics compared to a conditional knockout. In our model, conditional deletion of NCAM begins at approximately 2.5 weeks of postnatal life, meaning NCAM is still present during the critical early pre- and postnatal development and is further restricted to the hippocampus and forebrain (Bukalo et al., 2004). Consequently, the increase in exploration in the constitutive NCAM-KOs might be due to the gene deletion during early development and/or to the overall deletion of the gene throughout the brain, including regions crucially involved in locomotion. With respect to emotional and anxiety-related behavior, constitutive NCAMKOs were characterized with higher levels of anxiety in several tests, including the EPM, light-dark box and OF test (Aonurm-Helm et al., 2008). However, previous studies have shown that NCAM-cKO is associated with decreased anxiety in the elevated zero maze in animals under basal conditions (Bisaz et al., 2009), whereas we did not find differences concerning the time spent in the center of the open field under baseline conditions, but following stress. Further studies are necessary to clarify the differential impact of conditional and constitutive NCAM deletion together with stress exposure on anxiety-like behavior in mice. 


\section{basal}

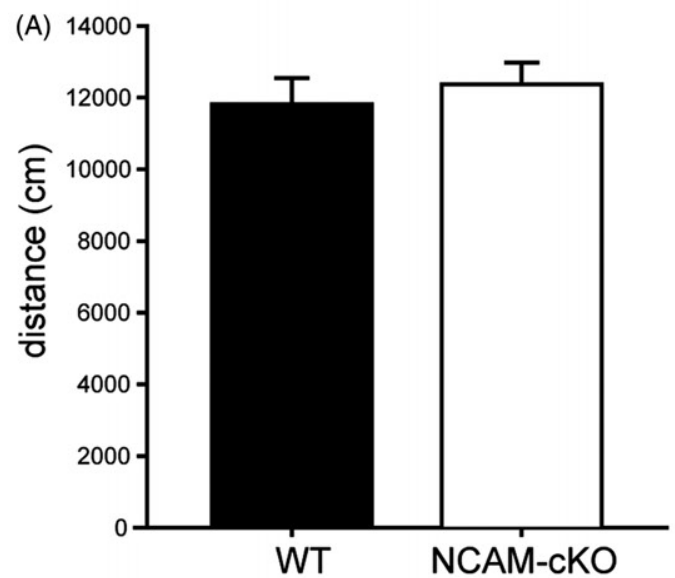

\section{subchronic}

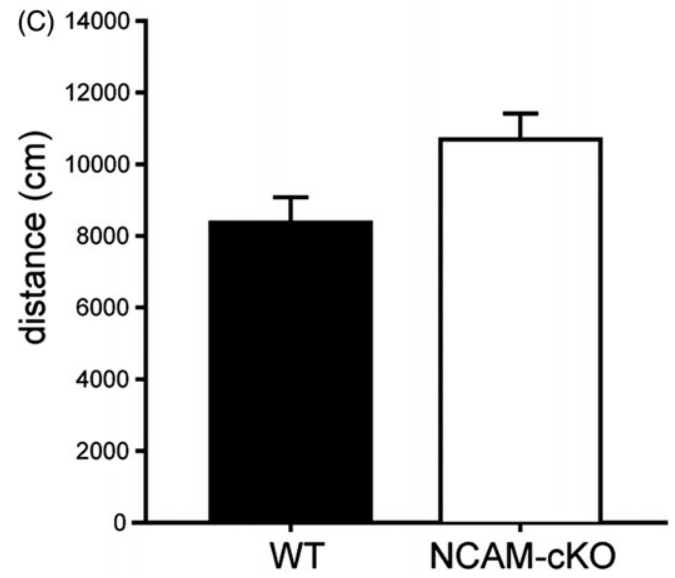

\section{chronic}

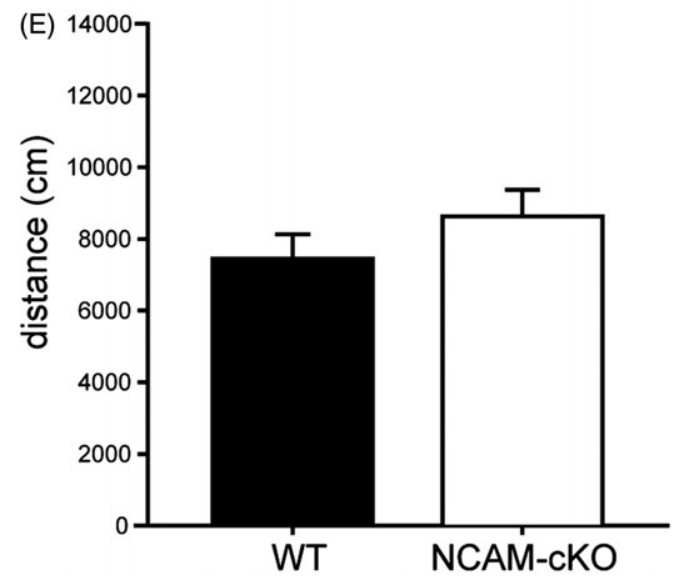

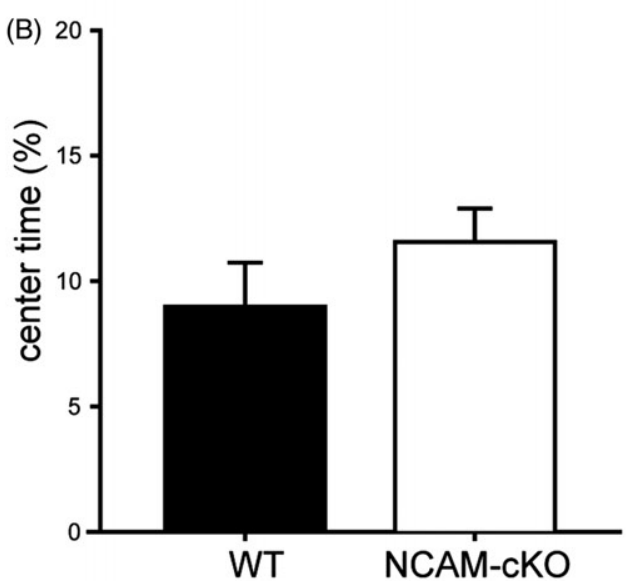
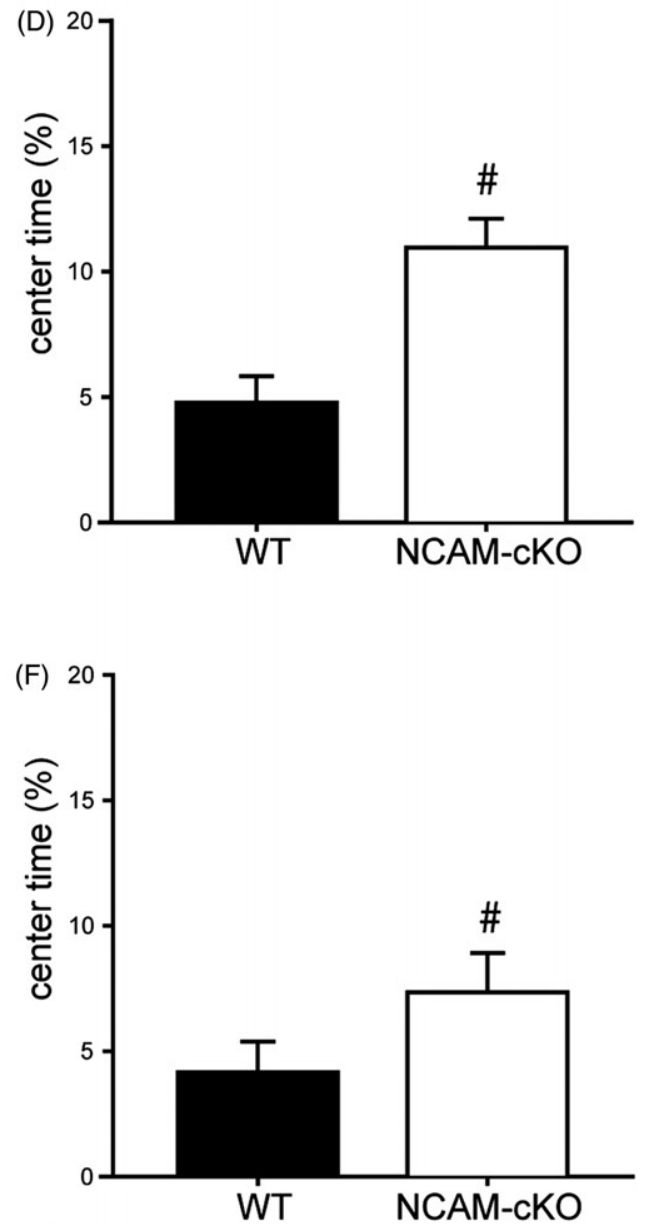

Figure 3. Open field behavior. (A-B) Experiment 1: Open field behavior under basal conditions. (A) Distance travelled in the open field. There were no differences in distance travelled between the genotypes. (B) Time in the center of the open field. The percentage of time spent in the center zone of the apparatus did not differ between the two groups under basal conditions. (C-F) Experiment 2. (C-D), Open field behavior after subchronic stress. (C) Distance travelled in the open field. No differences were detected in distance travelled between the genotypes. (D) Time in the center of the open field. The percentage of time spent in the center zone was significantly increased in NCAM-cKOs compared to WTs. (E-F) Open field behavior after chronic stress. (E) Distance travelled in the open field. After chronic stress, there was no significant difference between the two genotypes in distance travelled. (F) Time in the center of the open field. There was a significant genotype difference regarding percentage of time spent in the center zone. \#p<0.05 versus WT.

It has been suggested that some types of excessive aggression and violence can develop as a consequence of generally disturbed emotional regulation, such as abnormally high or low levels of anxiety (Haller et al., 2005, 2007; Neumann et al., 2010); thus, the effects observed in offensive behavior may be associated with the findings concerning emotionality. The consequences of chronic stress on aggression in NCAM-cKOs may be linked in a time- or duration-dependent manner to the development of emotional dysregulation. Whether these consequences may be present 

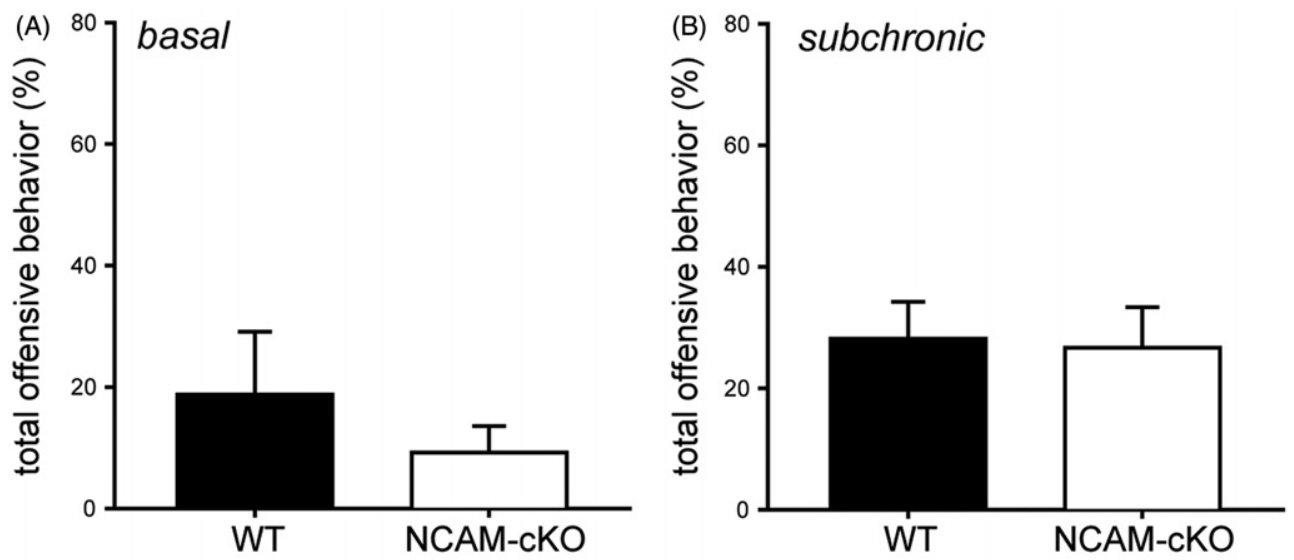

(C)

(D)
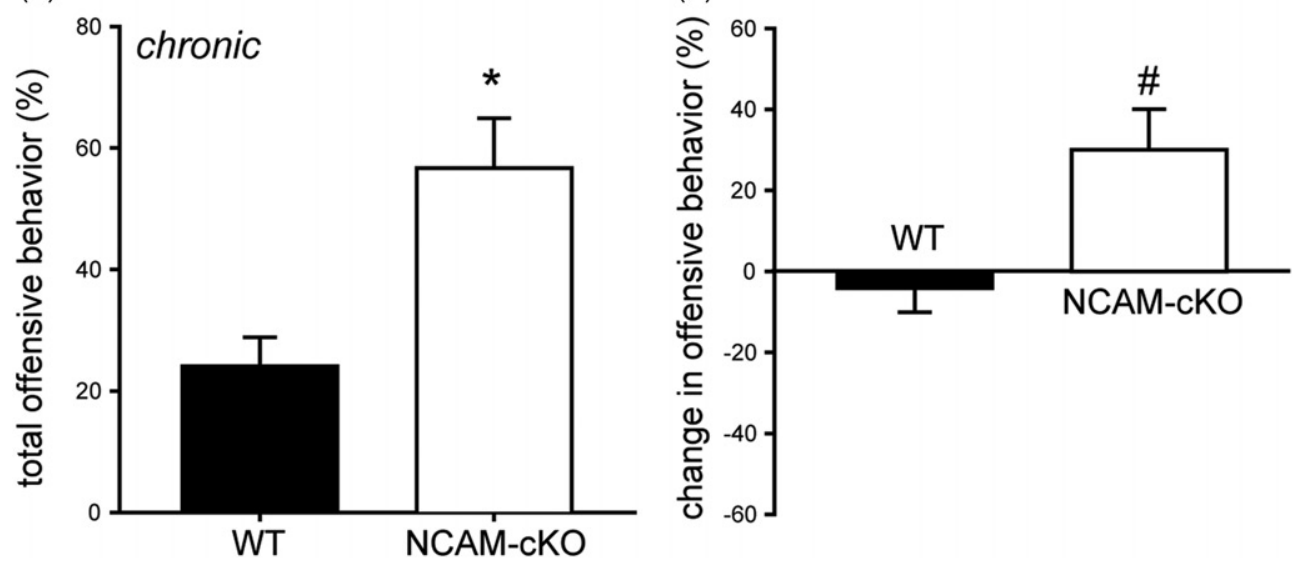

Figure 4. Aggressive-like behavior in the resident-intruder test. (A) Experiment 1: Aggressive behavior under basal conditions. No differences were observed between the two groups in the percentage of offensive behavior during the RI test. (B-D) Experiment 2. (B) Aggressive behavior after subchronic stress. The NCAM-cKOs did not differ from WTs regarding offensive behavior in the RI test after the first period of stress. (C) Aggressive behavior after chronic stress. We observed a significantly higher percentage of time of offensive behavior in NCAM-cKOs compared to WTs after the second period of stress. (D) Change in aggression-related behavior depending on the stress duration. When calculating the change in offensive behavior from RI1 to RI2, a significantly higher increase in offensive behavior in NCAM-cKOs compared to WTs was evident. * $p<0.01 \# p<0.05$ versus WT.

after prolonged stress remains a subject for further investigations. In addition, we cannot completely rule out a possible contribution of the group-housing, involving social conflicts, on aggressive behaviors (van Loo et al., 2001).

It has been postulated that there is a decline of the plasticity of emotional and aggressive behavior with increasing age, which is most evident in the inadequate response to environmental challenges (Dean, 1962; Sapolsky et al., 1986). As the animals in our experiments were not young adults but rather middle-aged, the possibility that age-related factors could have contributed to the observed vulnerability to stress warrants further investigation.

In summary, here we provide evidence of a conditional and forebrain-specific involvement of NCAM in the regulation of aggressive behavior dependent on the duration of environmental challenges. Future studies will be devoted to address possible structural alterations in the brains of stressed NCAM-cKO mice, which may underlie the observed behavioral phenotype.

\section{Declaration of interest}

This work was supported by grants from the European Union integrated project MEMSTICK (FP7-HEALTH-F2M-2007201600; MemStick), the Swiss National Science Foundation
(310000-120791 and 31003AB-135710; Sinergia CRSIK3122691; and the NCCR "The synaptic basis of mental diseases" as well as intramural funding from the EPFL.

\section{References}

Aonurm-Helm A, Jurgenson M, Zharkovsky T, Sonn K, Berezin V, Bock E, Zharkovsky A. (2008). Depression-like behaviour in neural cell adhesion molecule (NCAM)-deficient mice and its reversal by an NCAM-derived peptide, FGL. Eur J Neurosci 28:1618-28.

Bisaz R, Sandi C. (2011). Vulnerability of conditional NCAM-deficient mice to develop stress-induced behavioral alterations. Stress 15(2): 195-206.

Bisaz R, Schachner M, Sandi C. (2009). Causal evidence for the involvement of the neural cell adhesion molecule, NCAM, in chronic stress-induced cognitive impairments. Hippocampus 21:56-71.

Brennaman LH, Maness PF. (2010). NCAM in neuropsychiatric and neurodegenerative disorders. Adv Exp Med Biol 663:299-317.

Bukalo O, Fentrop N, Lee AYW, Salmen B, Law JWS, Wotjak CT, et al. (2004). Conditional ablation of the neural cell adhesion molecule reduces precision of spatial learning, long-term potentiation, and depression in the CA1 subfield of mouse hippocampus. J Neurosci 24: $1565-1577$.

Calandreau L, Marquez C, Bisaz R, Fantin M, Sandi C. (2010). Differential impact of polysialyltransferase ST8SiaII and ST8SiaIV knockout on social interaction and aggression. Genes Brain Behav 9: 958--67.

Caspi A, McClay J, Moffitt TE, Mill J, Martin J, Craig IW, Taylor A, Poulton R. (2002). Role of genotype in the cycle of violence in maltreated children. Science 297:851-4. 
Caspi A, Sugden K, Moffitt TE, Taylor A, Craig IW, Harrington H, McClay J, et al. (2003). Influence of Life Stress on Depression: moderation by a polymorphism in the 5-HTT gene. Science 301: 386-9.

Chekhonin VP, Shepeleva II, Gurina OI. (2008). Disturbances in the expression Of neuronal cell adhesion proteins NCAM: Clinical aspects. Neurochem J 2:239-51.

Conboy L, Bisaz R, Markram K, Sandi C. (2010). Role of NCAM in emotion and learning. Adv Exp Med Biol 663:271-96.

Cordero MI, Poirier GL, Marquez C, Veenit V, Fontana X, Salehi B, Ansermet F, Sandi C. (2012). Evidence for biological roots in the transgenerational transmission of intimate partner violence. Translational Psychiatry 2:e106.

Craig IW. (2007). The importance of stress and genetic variation in human aggression. Bioessays 29:227-36.

Craig IW, Halton KE. (2009). Genetics of human aggressive behaviour. Hum Genet 126:101-13.

Cunningham BA, Hemperly JJ, Murray BA, Prediger EA, Brackenbury R, Edelman GM. (1987). Neural cell adhesion molecule: structure, immunoglobulin-like domains, cell surface modulation, and alternative RNA splicing. Science 236:799-806.

Dahlhaus R, El-Husseini A. (2010). Altered neuroligin expression is involved in social deficits in a mouse model of the fragile $X$ syndrome. Behav Brain Res 208:96-105.

Dalva MB, McClelland AC, Kayser MS. (2007). Cell adhesion molecules: signalling functions at the synapse. Nat Rev Neurosci 8: 206-20.

Dean LR. (1962). Aging and the decline of affect. J Gerontol 17:440-6.

de Kloet ER, Joels M, Holsboer F. (2005). Stress and the brain: from adaptation to disease. Nat Rev Neurosci 6:463-75.

Frazzetto G, Di Lorenzo G, Carola V, Proietti L, Sokolowska E, Siracusano A, Gross C, Troisi A. (2007). Early trauma and increased risk for physical aggression during adulthood: the moderating role of MAOA genotype. PLoS One 2:e486.

Haller J, Kruk MR. (2006). Normal and abnormal aggression: human disorders and novel laboratory models. Neurosci Biobehav Rev 30: 292-303.

Haller J, Horvath Z, Bakos N. (2007). The effect of buspirone on normal and hypoarousal-driven abnormal aggression in rats. Progress NeuroPsychopharmacol Biol Psychiatry 31:27-31.

Haller J, Mikics A, Halasz J, Toth M. (2005). Mechanisms differentiating normal from abnormal aggression: glucocorticoids and serotonin. Eur J Pharmacol 526:89-100.

Hines RM, Wu L, Hines DJ, Steenland H, Mansour S, Dahlhaus R, Singaraja RR, et al. (2008). Synaptic imbalance, stereotypies, and impaired social interactions in mice with altered neuroligin 2 expression. J Neurosci 28:6055-67.

Kohl C, Riccio O, Grosse J, Zanoletti O, Fournier C, Schmidt MV, Sandi C. (2013). Hippocampal neuroligin-2 overexpression leads to reduced aggression and inhibited novelty reactivity in rats. PLoS One 8:e56871.

Lupien SJ, McEwen BS, Gunnar MR, Heim C. (2009). Effects of stress throughout the lifespan on the brain, behaviour and cognition. Nat Rev Neurosci 10:434-45.

Marquez C, Poirier GL, Cordero MI, Larsen MH, Groner A, Marquis J, Magistretti PJ, et al. (2013). Peripuberty stress leads to abnormal aggression, altered amygdala and orbitofrontal reactivity and increased prefrontal MAOA gene expression. Transl Psychiatry 3:e216.

McEwen BS. (2002). Sex, stress and the hippocampus: allostasis, allostatic load and the aging process. Neurobiol Aging 23:921-39.

Mineur YS, Prasol DJ, Belzung C, Crusio WE. (2003). Agonistic behavior and unpredictable chronic mild stress in mice. Behav Genet 33:513-19.

Moffitt TE. (2005). The new look of behavioral genetics in developmental psychopathology: gene-environment interplay in antisocial behaviors. Psychol Bulletin 131:533-54.

Neumann ID, Veenema AH, Beiderbeck DI. (2010). Aggression and anxiety: social context and neurobiological links. Front Behav Neurosci 4:12.

Newman TK, Syagailo YV, Barr CS, Wendland JR, Champoux M, Graessle M, Suomi SJ, et al. (2005). Monoamine oxidase A gene promoter variation and rearing experience influences aggressive behavior in rhesus monkeys. Biol Psychiatry 57:167-72.

Nunes PM, Wenzel A, Borges KT, Porto CR, Caminha RM, de Oliveira IR. (2009). Volumes of the hippocampus and amygdala in patients with borderline personality disorder: a meta-analysis. J Personality Disorders 23:333-45.

Poltorak M, Hemperly JJ, Williams JR, El-Mallakh R, Freed WJ. (1995). Disturbances in cell recognition molecules (N-CAM and L1 antigen) in the CSF of patients with schizophrenia. Exp Neurol 131: 266-72.

Rutter M, Moffitt TE, Caspi A. (2006). Gene-environment interplay and psychopathology: multiple varieties but real effects. J Child Psychol Psychiatry 47:226-61.

Rutter M, Silberg J. (2002). Gene-environment interplay in relation to emotional and behavioral disturbance. Annu Rev Psychol 53:463-90.

Sandi C. (1998). The role and mechanisms of action of glucocorticoid involvement in memory storage. Neural Plast 6:41-52.

Sandi C. (2004). Stress, cognitive impairment and cell adhesion molecules. Nat Rev Neurosci 5:917-30.

Sandi C, Merino JJ, Cordero MI, Touyarot K, Venero C. (2001). Effects of chronic stress on contextual fear conditioning and the hippocampal expression of the neural cell adhesion molecule, its polysialylation, and L1. Neuroscience 102:329-39.

Sandi C, Woodson JC, Haynes VF, Park CR, Touyarot K, LopezFernandez MA, Venero C, Diamond DM. (2005). Acute stressinduced impairment of spatial memory is associated with decreased expression of neural cell adhesion molecule in the hippocampus and prefrontal cortex. Biol Psychiatry 57:856-64.

Sandi C, Bisaz R. (2007). A model for the involvement of neural cell adhesion molecules in stress-related mood disorders. Neuroendocrinology 85(3):158-76.

Sapolsky RM, Krey LC, McEwen BS. (1986) The neuroendocrinology of stress and aging: the glucocorticoid cascade hypothesis. Endocr Rev 7:284-301.

Shapiro L, Love J, Colman DR. (2007). Adhesion molecules in the nervous system: structural insights into function and diversity. Annu Rev Neurosci 30:451-74.

Stork O, Welzl H, Cremer H, Schachner M. (1997). Increased intermale aggression and neuroendocrine response in mice deficient for the neural cell adhesion molecule (NCAM). Eur J Neurosci 9:1117-25.

Stork O, Welzl H, Wolfer D, Schuster T, Mantei N, Stork S, Hoyer D, et al. (2000). Recovery of emotional behaviour in neural cell adhesion molecule (NCAM) null mutant mice through transgenic expression of NCAM180. Eur J Neurosci 12:3291-306.

Stork O, Welzl H, Wotjak CT, Hoyer D, Delling M, Cremer H, Schachner M. (1999). Anxiety and increased 5-HT1A receptor response in NCAM null mutant mice. J Neurobiol 40:343-55.

van Loo PL, Mol, JA, Koolhaas JM, van Zutohen BF, Baumans V. (2001). Modulation of aggression in male mice: influence of group size and cage size. Physiol Behav 72(5):675-83.

Vawter MP, Howard AL, Hyde TM, Kleinman JE, Freed WJ. (1999). Alterations of hippocampal secreted N-CAM in bipolar disorder and synaptophysin in schizophrenia. Mol Psychiatry 4:467-75.

Veenema AH, Neumann ID. (2007). Neurobiological mechanisms of aggression and stress coping: a comparative study in mouse and rat selection lines. Brain Behav Evol 70:274-85.

Venero C, Tilling T, Hermans-Borgmeyer I, Schmidt R, Schachner M, Sandi C. (2002). Chronic stress induces opposite changes in the mRNA expression of the cell adhesion molecules NCAM and L1. Neuroscience 115:1211-19.

Wagner KV, Hartmann J, Mangold K, Wang XD, Labermaier C, Liebl C, Wolf M, et al. (2013). Homer1 mediates acute stress-induced cognitive deficits in the dorsal hippocampus. J Neurosci 33(9): 3857-64.

Wingenfeld K, Spitzer C, Rullkötter N, Löwe B. (2010). Borderline personality disorder: hypothalamus pituitary adrenal axis and findings from neuroimaging studies. Psychoneuroendocrinology 35:154-70.

Wood GE, Norris EH, Waters E, Stoldt JT, McEwen BS. (2008). Chronic immobilization stress alters aspects of emotionality and associative learning in the rat. Behav Neurosci 122:282-92.

Wood GE, Young LT, Reagan LP, McEwen BS. (2003). Acute and chronic restraint stress alter the incidence of social conflict in male rats. Horm Behav 43:205-13. 